\title{
MERS differs from SARS, say experts
}

A

new coronavirus with a high death rate and human-tohuman transmission. Cases emerging in travellers far from the disease's origin, including one in Indiana. It sounds like severe acute respiratory syndrome (SARS), but international researchers studying Middle East respiratory syndrome (MERS) say this new infection differs from SARS in important ways.

"I would not say it is like SARS," says Arnaud Fontanet, a principal investigator with the Pasteur Institute in Paris, France. But he offers a caveat: "It may become like SARS if the virus adapts to humans."

When SARS spread to humans from civets (a cat-like mammal), Fontanet says it underwent two genetic mutations that allowed it to target a receptor in humans, making it much more infective and leading to rapid spread. To date, that doesn't appear to have happened with MERS. Fontanet says the spread of MERS is below the tipping point that can lead to a pandemic. Further, a new study in mBio shows that the virus is genetically the same as another virus that is widespread and prevalent in camels, suggesting that it has not yet adapted to specifically target humans. As well, Fontanet says the virus appears to target a different receptor in mammals, including humans, than the one for SARS.

In a July 2013 paper published in The Lancet, Fontanet and his team showed that MERS' transmissibility from human-to-human was low, and in fact lower than prepandemic SARS.

What is "worrisome," says Fontanet, is the recent acceleration in MERS cases. In April alone there were 145 cases, or $56 \%$ of the total to date; all were in Saudi Arabia and the United Arab Emirates. As of Apr. 26, the World Health Organization (WHO) reported a total of 261 laboratoryconfirmed cases and 93 deaths. According to $\mathrm{WHO}, 75 \%$ of recent cases appear to be secondary (human-to-

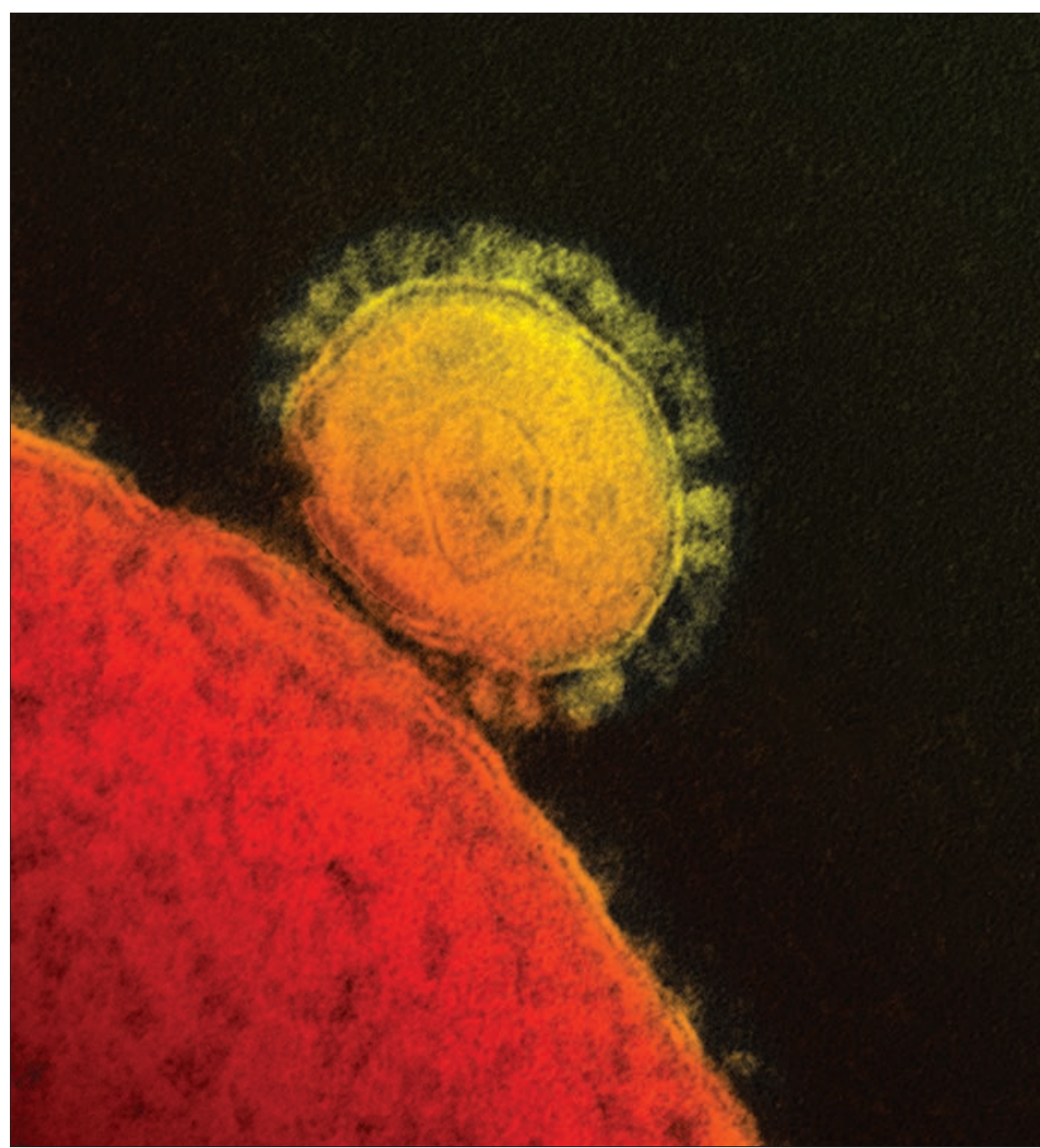

The first case of MERS in North America has raised alarm about a possible pandemic.

human) infections. (These figures do not include one new case in Egypt and the one in the United States.)

How MERS is transmitted remains unclear. The $m B i o$ study found antibodies to the MERS coronavirus in $74 \%$ of 203 dromedary camels in five disparate regions of Saudi Arabia. Thus it seems likely that camels are the source of infection, but that's not the end of the story.

"If the virus easily jumps directly from camels to humans, one would expect that people interacting intensely with camels represent a high proportion of cases, but that's not what we are seeing," say authors Abdulaziz Alagaili, Mammals Research Chair at King Saud
University in Saudi Arabia, and Thomas Briese of the Mailman School of Public Health, Columbia University, New York, in an email. "We are now investigating potential routes for human infection through exposure to camel milk or meat products."

They are also investigating other domestic animals, including rodents and bats, to discover whether there is another animal reservoir. Their study had tested domestic sheep and goats as well, and found no infection.

The researchers also found the virus in archived blood samples from camels, suggesting that the virus or a similar one has circulated in camels for two decades. The mystery is why human 
cases have surfaced in only in the last two years.

"It may well be that MERS occurred much earlier than 2012 in humans, but was not recognized," say the authors. "One would need to find specimens from earlier suspect cases and demonstrate the presence of the virus. Alternatively, the virus may have changed recently to cause human disease."

Another recent study in The Lancet Infectious Diseases modelled how the virus could spread during air travel, and found a small but real risk of spread from those infected during flight. The risk ranged from one new infection in a five-hour flight in first class, to 15 infections from a "superspreader" (a highly contagious carrier) travelling 13 hours in economy. Those infected would probably show no signs when disembarking, says Brian Coburn, a postdoctoral fellow at the Centre for Biomedical Modeling at the University of California Los Angeles. "These newly infected individuals may be difficult to identify and could cause new outbreaks."
Authors of all these papers stress the need for public health measures.

"Prevention of human infection will be important not only to prevent illness, but also to limit opportunities for virus adaptation and selection in the human host," say Alagaili and Briese. They suggest people avoid unprotected exposure to camels and that a vaccine be developed not only for humans but also for the camel hosts. - Carolyn Brown, Ottawa, Ont.

CMAJ 2014. DOI:10.1503/cmaj.109-4798 